Article

\title{
Slow-Binding Inhibition of Tyrosinase by Ecklonia cava Phlorotannins
}

\author{
Jang Hoon Kim ${ }^{1,2,+} \mathbb{D}$, Sunggun Lee ${ }^{1,+}$, Saerom Park ${ }^{1}$, Ji Soo Park ${ }^{1}$, Young Ho Kim ${ }^{1, *}$ and \\ Seo Young Yang ${ }^{1, *}$ \\ 1 College of Pharmacy, Chungnam National University, Daejeon 34134, Korea; oasis5325@gmail.com (J.H.K.); \\ staroot99@naver.com (S.L.); tofhadlzz@naver.com (S.P.); bjs442@naver.com (J.S.P.) \\ 2 Biospectrum Life Science Institute, A-1805, U-TOWER, 767, Sinsu-ro, Suji-gu, Yongin-si, \\ Gyeonggi-do 16827, Korea \\ * Correspondence: yhk@cnu.ac.kr (Y.H.K.); syyang@cnu.ac.kr (S.Y.Y.); \\ Tel.: +82-42-821-5933 (Y.H.K.); +82-42-821-7321 (S.Y.Y.) \\ + These authors contributed equally to this work.
}

Received: 29 May 2019; Accepted: 14 June 2019; Published: 16 June 2019

check for

\begin{abstract}
Tyrosinase inhibitors improve skin whitening by inhibiting the formation of melanin precursors in the skin. The inhibitory activity of seven phlorotannins (1-7), triphlorethol A (1), eckol (2), 2-phloroeckol (3), phlorofucofuroeckol A (4), 2-O-(2,4,6-trihydroxyphenyl)-6,6'-bieckol (5), 6,8'-bieckol (6), and 8,8'-bieckol (7), from Ecklonia cava was tested against tyrosinase, which converts tyrosine into dihydroxyphenylalanine. Compounds 3 and 5 had $\mathrm{IC}_{50}$ values of $7.0 \pm 0.2$ and $8.8 \pm 0.1$ $\mu \mathrm{M}$, respectively, in competitive mode, with $K_{\mathrm{i}}$ values of $8.2 \pm 1.1$ and $5.8 \pm 0.8 \mu \mathrm{M}$. Both compounds showed the characteristics of slow-binding inhibitors over the time course of the enzyme reaction. Compound 3 had a single-step binding mechanism and compound 5 a two-step-binding mechanism. With stable AutoDock scores of -6.59 and $-6.68 \mathrm{kcal} / \mathrm{mol}$, respectively, compounds 3 and 5 both interacted with His85 and Asn260 at the active site.
\end{abstract}

Keywords: Ecklonia cava; Laminareaceae; phlorotannins; competitive inhibitor; slow binding inhibitor

\section{Introduction}

Melanin is produced in skin epidermal cells to protect the skin from ultraviolet radiation [1]. The overproduction and accumulation of melanin causes age spots, freckles, melisma, and hyperpigmentation [1,2]. Tyrosinase (EC 1.14.18.1), which belongs to the type 3 copper protein family, is a key enzyme in melanogenesis [1,3]. It is a multifunctional oxide that contains a copper in each of two sets of three histidine residues in the active site. [4]. Furthermore, it catalyzes the hydroxylation of 1-tyrosine to 1-3,4-dihydroxyphenylalanine (1-DOPA), and the subsequent oxidation of 1-DOPA to DOPA quinone [3,5]. These products are used in melanin biosynthesis [4]. Recently, the tyrosinase inhibitors kojic acid and arbutin were developed to improve skin whitening and as anti-hyperpigmentation agents [3,6]. However, they had undesirable side effects, including dermatitis, skin irritation, and DNA damage $[1,4]$. Much research has examined a variety of natural plants, with the aim of developing new inhibitors [7]. This study also sought alternative inhibitors without adverse effects.

Ecklonia cava, in the family Laminariaceae, grows along the coast of Jeju Island, Korea [8] and is widely used in Korea as a food ingredient, animal feed, and medicine [9]. E. cava contains phlorotannins in polymerized phloroglucinol units [8]. Phlorotannins are secondary metabolites that are important to human health [10]. These compounds have antioxidant, anti-cancer, anti-allergy, and anti-HIV bioactivities [10,11]. In addition, the compounds eckol, phlorofucofureckol A, and dieckol, from the edible brown alga Ecklonia stolonifera, exhibit anti-tyrosinase activity at macromolar concentrations [12]. 
This study evaluated the tyrosinase inhibitory activity of minor components from E. cava. Among seven isolated compounds (1-7), two minor compounds ( 3 and 5 ) had $\mathrm{IC}_{50}$ values of less than $10 \mu \mathrm{M}$, and also exhibited competitive and slow-binding inhibition of tyrosinase. This study also shows how these compounds interact with the catalytic site of tyrosinase via molecular docking.

\section{Results and Discussion}

\subsection{Isolation and Identification}

The ethyl acetate fraction of ethanol extract of E. cava was known to suppress the catalytic reaction of tyrosinase [13]. Phlorotannins, eckol, triphlorethol-A, phlorofucofuroeckol A, and dieckol, were reported to be isolated from this fraction $[14,15]$. To isolate phlorotannins which have not been identified as tyrosinase inhibitors, dried E. cava powder $(1.1 \mathrm{~kg})$ was extracted by reflux with $80 \%$ ethanol for $72 \mathrm{~h}$. The concentrated $(80 \%)$ ethanol extract $(290.0 \mathrm{~g})$ was progressively divided into $n$-hexane, ethyl acetate, and butanol layers. The ethyl acetate layer (54.9 g) was subjected to open column chromatography with silica gel and C-18 resin to obtain seven phlorotannins (1-7). By comparing spectroscopic data with reported results, the compounds were identified as triphlorethol A (1) [16], eckol (2) [8], 2-phloroeckol (3) [16], phlorofucofuroeckol A (4) [12], 2-O-(2,4,6-trihydroxyphenyl)-6,6'-bieckol (5) [17], 6,8' -bieckol (6) [18], and 8,8'-bieckol (7) [8] (Figure 1).<smiles>Oc1cc(O)cc(Oc2c(O)cc(O)cc2Oc2c(O)cc(O)cc2O)c1</smiles>

1

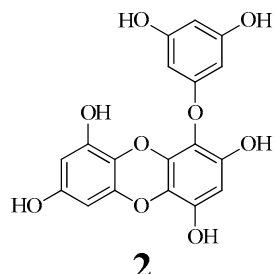

2

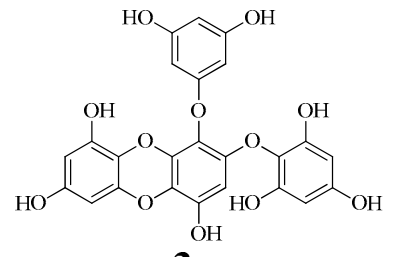

3

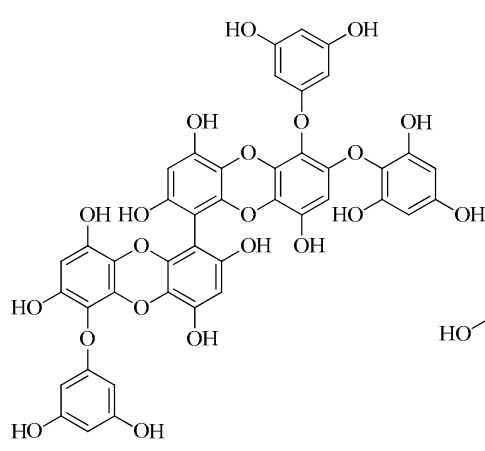

5<smiles>OC1=COc2c3oc(c4cc(O)c(-c5c(O)cc(O)c6c5Oc5c(O)cc(O)c(Oc7cc(O)cc(O)c7)c5O6)c(O)c4oc2c(O)cc3O)=CC(O)=C1</smiles>

6<smiles></smiles>

4<smiles></smiles>

7

Figure 1. Structure of compounds 1-7 from E. cava.

\subsection{Inhibition of Phlorotannins on Tyrosinase}

Phlorotannins of E. cava and E. stolonifera were reported to have the inhibitory activity on mushroom tyrosinase [12,19]. 7-phloroeckol and dieckol were revealed to be the potential inhibitors within micromole concentration $[13,19]$. This study evaluated the ability of the isolated phlorotannins 1-7 to suppress the catalytic reaction of tyrosinase over time, in the absence or presence of inhibitor. Their inhibitory activity was calculated using equation (1). A commercial tyrosinase inhibitor was used as a positive control (kojic acid; $\mathrm{IC}_{50}=25.0 \pm 0.4 \mu \mathrm{M}$ ). To identify potent inhibitors, the inhibitory activity of all of the isolated compounds at $100 \mu \mathrm{M}$ was tested against tyrosinase in vitro (Table 1). Of these, compounds $\mathbf{2}-\mathbf{5}$ were confirmed to have inhibitory activity exceeding $\mathbf{5 0} \%$. Serial dilutions were used to calculate the $\mathrm{IC}_{50}$ values. The tyrosinase inhibitory activity increased in a dose-dependent fashion (Figure 2A). Compounds $\mathbf{2}-\mathbf{5}$ showed inhibitory activity, with $\mathrm{IC}_{50}$ values of $7.0 \pm 0.2$ to 
$66.4 \pm 0.1 \mu \mathrm{M}$ (Table 1). Of these, compounds 3 and 5 had inhibitory activity at $<10 \mu \mathrm{M}$. Interestingly, the structure of compound 5 contained the moiety of compound 3.

Our studies and reported results showed that phlorotannins, 2-phloroeckol, 7-phloroeckol dieckol, and 2-O-(2,4,6-trihydroxyphenyl)-6,6'-bieckol, substituted with phloroglucinol in eckol increased the inhibitory effect on tyrosinase compared to other phlorotannins [12,19]. Recently, secondary metabolites from terrestrial plants have been studied primarily for tyrosinase inhibitors [4-7]. Morin, norartocarpetin, artogomezianone, and (-)- $\mathrm{N}$-formylanonaine have been revealed as the potential inhibitors [4-7]. Interest in the components from seaweed led to the study of the isolation of phlorotannin. It has been proven that they improve skin whitening $[12,13,19]$. These results suggest that 2-phloroeckol and 2-O-(2,4,6-trihydroxyphenyl)-6,6'-bieckol are tyrosinase inhibitors that can replace phenolic compounds [4-7].

To gain insight into the interaction of the enzyme with phlorotannins, as tyrosinase inhibitors, the products of the catalytic reaction were determined by ultraviolet-visible photometry after the inhibitor was added to the enzyme solution. The $\mathrm{IC}_{50}$ values of compounds $\mathbf{3}$ and $\mathbf{5}$ increased linearly with [S]/Km (Figure 2B). Furthermore, compounds 3 and $\mathbf{5}$ were produced with a set of the family liners by various inhibitors at substrate concentrations ranging from 0.156 to $2.50 \mathrm{mM}$. As shown in Lineweaver-Burk plots (Figure 2C,D), they had different values of $-1 / \mathrm{Km}$ and the same $1 / \mathrm{Vmax}$. The interactions with tyrosinase were competitive, with respective $K_{i}$ values of $8.2 \pm 1.1$ and $5.8 \pm$ $0.8 \mu \mathrm{M}$ based on Dixon plots (Figure 2E,F, Table 1). Phlorotannins from seaweeds have been used to develop the non-competitive tyrosinase inhibitors 7-phloroeckol, dieckol, and phlorofucofuroeckol A, and the competitive inhibitors phloroglucinol and eckstolonol $[12,13]$. We found that compounds 3 and 5 competitively inhibited the catalysis of tyrosinase and might be useful for improving whitening.

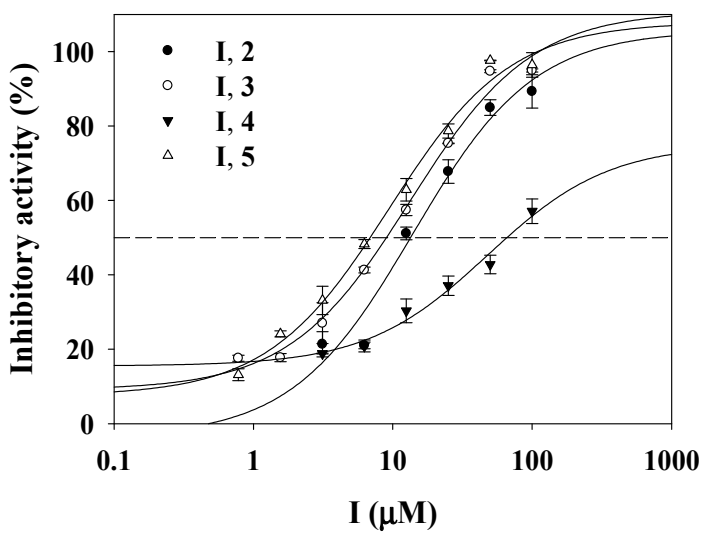

A

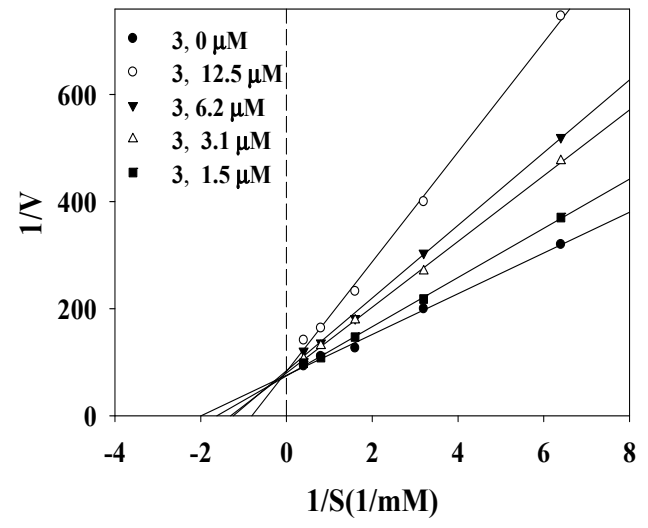

C

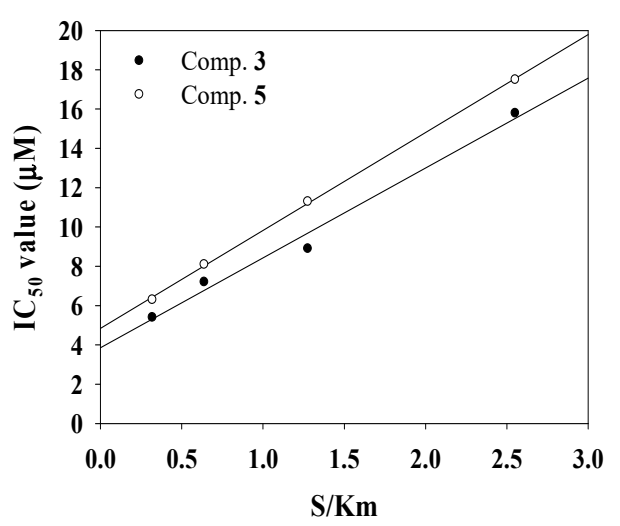

B

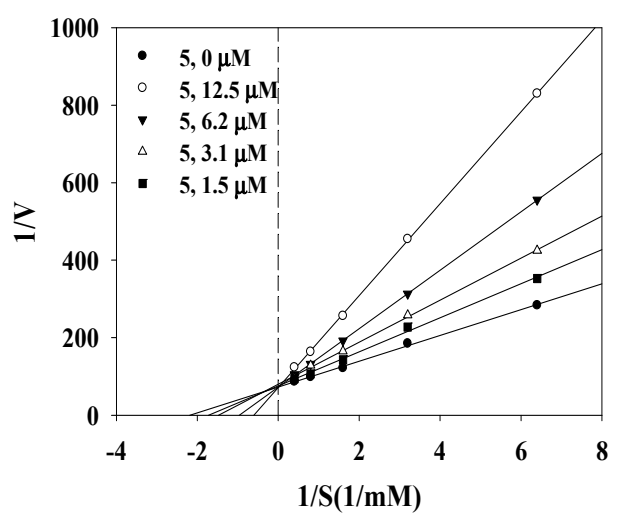

D

Figure 2. Cont. 

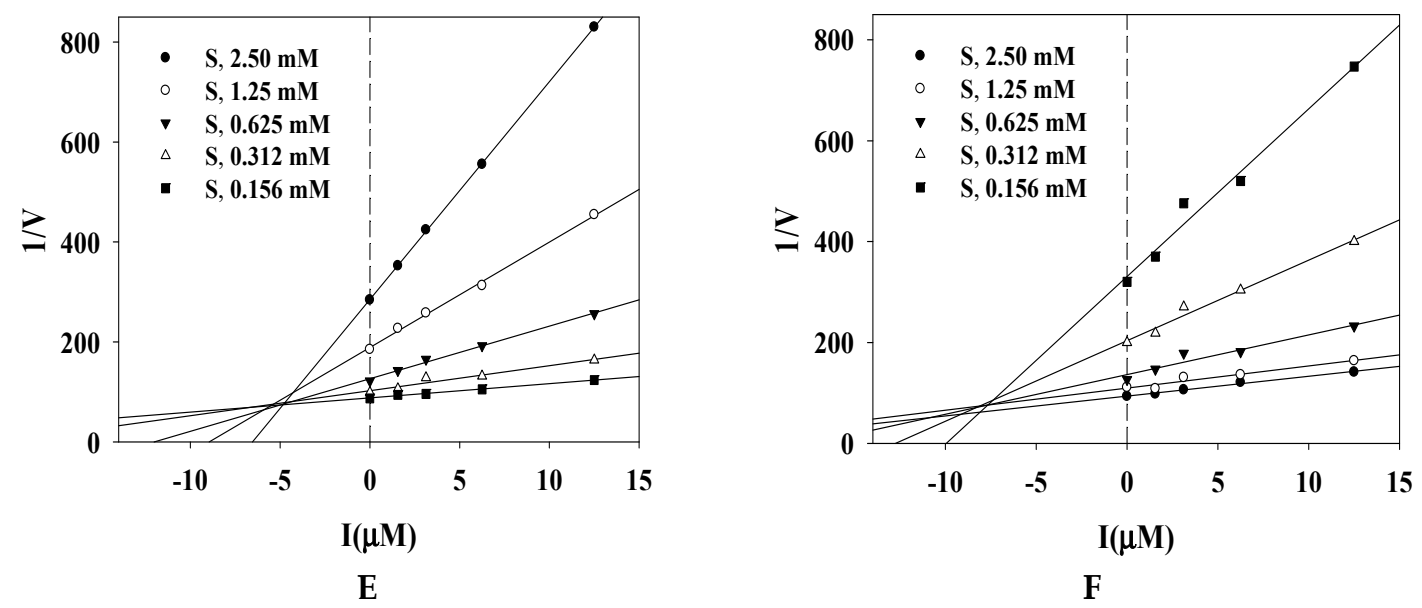

Figure 2. Inhibitory activity of compounds on tyrosinase (A). Effects of $\mathrm{S} / \mathrm{Km}$ ratio on the $\mathrm{IC}_{50}$ values (B). Lineweaver-Burk (C,D) and Dixon (E,F) plots of tyrosinase inhibition by compounds, respectively.

Table 1. Tyrosinase inhibitory activities of compounds 1-7.

\begin{tabular}{|c|c|c|c|}
\hline & \multicolumn{3}{|c|}{ Inhibitory Activity of Compounds on Tyrosinase ${ }^{a}$} \\
\hline & $100 \mu \mathrm{M}(\%)$ & $\mathrm{IC}_{50}(\mu \mathrm{M})$ & Binding Mode $\left(K_{i}, \mu \mathrm{M}\right)$ \\
\hline 1 & $26.7 \pm 1.0$ & N.T. ${ }^{c}$ & N.T. ${ }^{c}$ \\
\hline 2 & $86.0 \pm 4.7$ & $13.5 \pm 0.1$ & Reported as non-competitive ${ }^{d}$ \\
\hline 3 & $94.7 \pm 1.4$ & $7.0 \pm 0.2$ & Competitive $(8.2 \pm 1.1)$ \\
\hline 4 & $57.1 \pm 3.3$ & $66.4 \pm 0.1$ & N.T. ${ }^{c}$ \\
\hline 5 & $86.9 \pm 0.9$ & $8.8 \pm 0.1$ & Competitive $(5.8 \pm 0.8)$ \\
\hline 6 & $41.2 \pm 4.3$ & N.T. ${ }^{c}$ & N.T. ${ }^{c}$ \\
\hline 7 & $43.3 \pm 1.5$ & N.T. ${ }^{c}$ & N.T. ${ }^{c}$ \\
\hline
\end{tabular}

Kojic acid $^{\mathrm{b}} \quad 25.0 \pm 0.4$

a All compounds examined in a set of triplicated experiment; ${ }^{\mathrm{b}}$ Positive control; ${ }^{\mathrm{c}}$ Not tested; ${ }^{\mathrm{d}}$ Reference [12]

\subsection{Slow-Binding Inhibition}

To confirm time-dependent inhibition by the potential inhibitors (compounds 3 and 5 ), the substrate was added into a mixture of ligand $(3.1 \mu \mathrm{M})$ that was preincubated with tyrosinase. Over time, their inhibitory activities increased. To calculate the slow-binding parameters $\left(k_{3}, k_{4}, k_{5}, k_{6}\right.$, and $\left.k_{\text {app }} i\right)$, the progress curves were analyzed using equation (2), with increasing concentrations of compounds 3 and 5 (Figure 3C,D); a replot of $k_{\text {obs }}$ was obtained as [I] (Figure 3E,F). The replot of compound 3 was a straight line that fit equation (3). When forming an encounter complex (EI) of the receptor with the ligand, compound 3 slowly binds to the active site of tyrosinase according to slow-binding mechanism A (a single-step binding mechanism) (Figure 3E) [20]. In comparison, the replot of $k_{\text {obs }}$ for compound 5 fit a hyperbolic equation (5) based on mechanism B (a two-step binding mechanism). This indicates that enzyme isomerization ( $\left.E^{*} \mathrm{I}\right)$ results in slow bonding after the ligand rapidly interacts with the receptor [20,21]. Table 2 shows the kinetic parameters for the time-dependent inhibition of tyrosinase by the inhibitors. The two strongest inhibitors had different slow-binding mechanisms. Inhibitor $\mathbf{5}$, which has a higher molecular weight, induced a new conformational state of the enzyme.

Table 2. Kinetics parameters of tyrosinase by compounds $\mathbf{3}$ and $\mathbf{5}$.

\begin{tabular}{cccccc}
\hline Compound & $K_{\mathbf{3}}\left(\mathrm{mMs}^{-\mathbf{1}}\right)$ & $k_{\mathbf{4}}\left(\mathrm{s}^{-\mathbf{1}}\right)$ & $\boldsymbol{K}_{\mathbf{5}}\left(\mathrm{s}^{-\mathbf{1}}\right)$ & $\boldsymbol{K}_{\mathbf{6}}\left(\mathrm{s}^{-\mathbf{1}}\right)$ & $k$ app $\boldsymbol{i}(\boldsymbol{\mu M})$ \\
\hline $\mathbf{3}$ & 0.0002 & 0.0013 & - & - & $6.5 \mu \mathrm{M}$ \\
$\mathbf{5}$ & - & - & 0.0047 & 0.0003 & $4.4 \mu \mathrm{M}$ \\
\hline
\end{tabular}




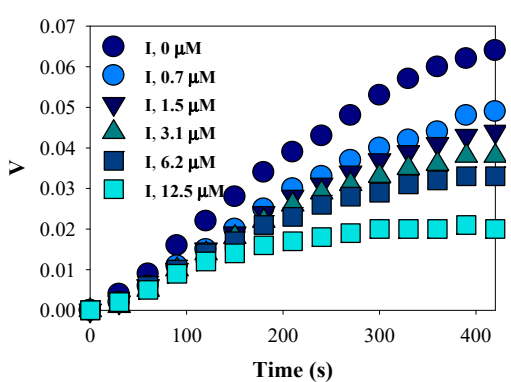

A

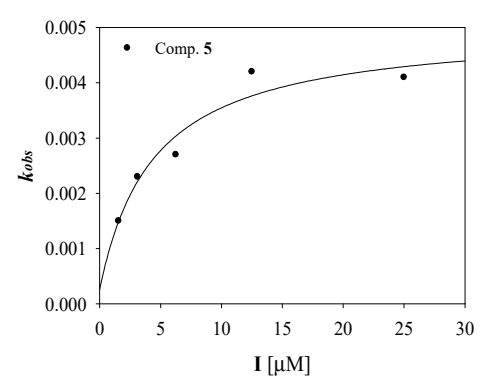

D

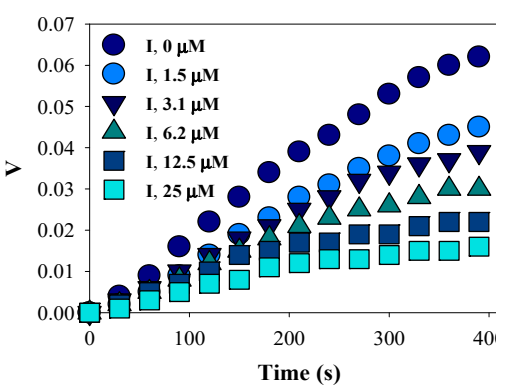

B

\section{Slow binding Mechanism A}

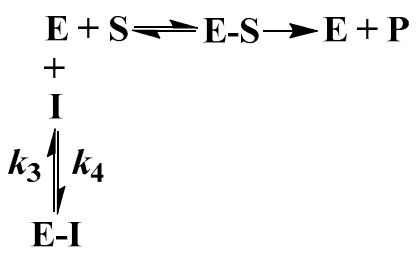

E

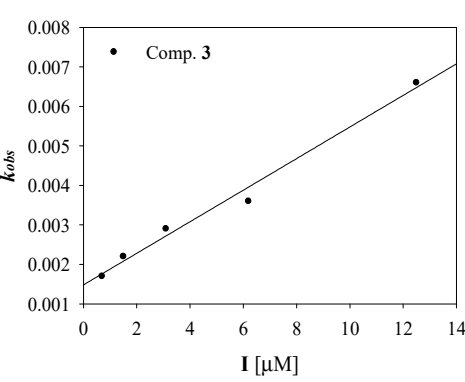

C

Mechanism B

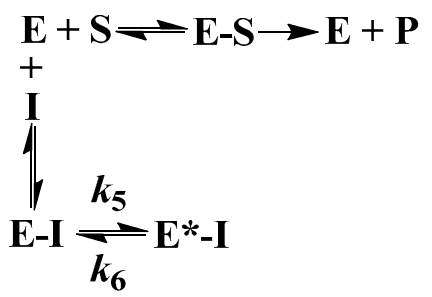

F

Figure 3. Progress curves for slow-binding inhibition (A,B) and dependence of the values of $k_{\mathrm{obs}}$ on the concentration of compounds 3 and $\mathbf{5}$ (C,D) of tyrosinase. Mechanism of slow binding inhibitor (E,F).

\subsection{Molecular Docking}

Molecular docking was performed to determine how the ligand interacts with the active site of tyrosinase. The best fit of their complex was considered to have the lowest energy, as calculated using Autodock 4.2. As indicated in Figure 4A-E and Table 3, the AutoDock scores of compounds 3 and 5 showed a trend similar to their $\mathrm{IC}_{50}$ values in vitro. They covered the hole and area around the active site, with calculated energies of -6.59 and $-6.68 \mathrm{kcal} / \mathrm{mol}$, respectively (Figure $4 \mathrm{~A}$ ). Compound 3 was held by six hydrogen bonds with three amino acids (Glu256 2.82 $\AA$, Asn260 $2.73 \AA$, and Met280 $2.68 \AA$ ) and hydrophobic interactions with 15 amino acids (Figure 4B,C, Table 3). The phloroglucinol moiety of compound 3 had a $\pi-\pi$ bond interaction with His 85 at $9.4 \AA$ distance (Figure $4 \mathrm{E}$ ). For compound

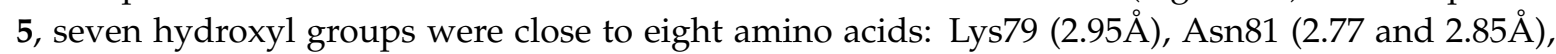

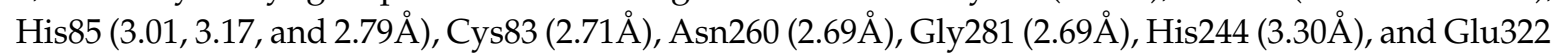

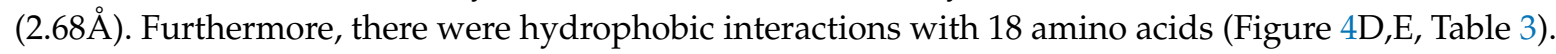

Table 3. Hydrogen bonding interactions and Autodock score between tyrosinase and inhibitors

\begin{tabular}{ccc}
\hline Compound & Hydrogen Bonds (̊̊) & Binding Energy (kcal/mol) \\
\hline 3 & Glu256(2.82), Asn260(2.73), Met280(2.68) & -6.59 \\
& Lys79(2.95), Asn81(2.85), 2.77), Cys83(2.71), & -6.68 \\
5 & His85(2.77, 3.17, 2.79), His244(3.30), Asn260(2.69), & \\
& Gly281(2.69), Glu322(2.68) & \\
\hline
\end{tabular}



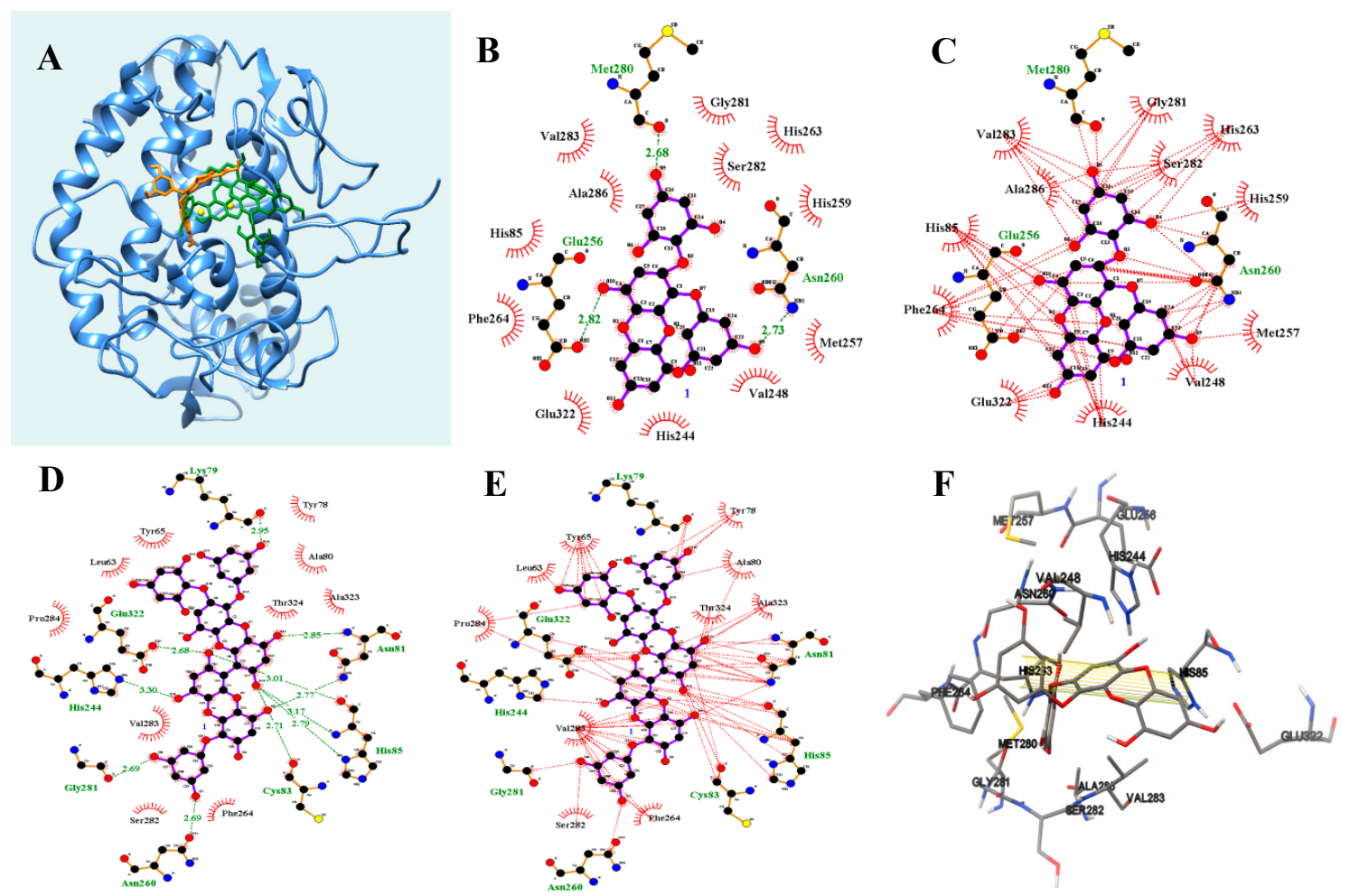

Figure 4. The binding pose (A) of two compounds 3 and 5 into tyrosinase (orange: 3 , green: 5). Hydrogen bonds (B,C), hydrophobic interactions (D,E), and $9.4 \AA \pi-\pi$ interaction (F) of inhibitor with the enzyme.

\section{Materials and Methods}

\subsection{General Experimental Procedures}

NMR experiments were conducted on an ECA500 (JEOL, Tokyo, Japan), with the chemical shift referenced to the residual solvent signals, using methanol- $d_{4}$ and DMSO- $d_{6}$ as a solvent. TLC analysis was performed on silica-gel $60 \mathrm{~F}_{254}$ and RP-18 $\mathrm{F}_{254 \mathrm{~S}}$ plates (both $0.25 \mathrm{~mm}$ layer thickness, Merck, Darmstadt, Germany). Compounds were visualized by dipping plates into $10 \%(v / v) \mathrm{H}_{2} \mathrm{SO}_{4}$ reagent and then air heat treated at $300{ }^{\circ} \mathrm{C}$ for $15 \mathrm{~s}$. Silica gel (Merck 60A, 70-230 or 230-400 mesh ASTM) and reversed-phase silica gel (YMC Co., ODS-A $12 \mathrm{~nm} \mathrm{S-150,} \mathrm{S-75} \mu \mathrm{m}$ ) were used for open column chromatography. Tyrosinase (T3824), kojic acid (K3125) and L-tyrosine (T3754) were purchased from Sigma-Aldrich (ST. Louis, Mo, USA). UV-vis and fluorescence spectra were measured by TECAN infinite $200 \mathrm{PRO}^{\circledR}$ spectrophotometer (Zurich, Switzerland).

\subsection{Plant Material}

E. cava was purchased from a herbal market in Jeju Island, Korea, on May 2015. One of the author (Prof. Y.H. Kim) identified this brown algal species. A voucher specimen (CNU-15005) was deposited at the Herbarium, College of Pharmacy, Chungnam National University (CNU).

\subsection{Extraction and Isolation}

The dried powder $(1.0 \mathrm{~kg})$ of E. cava was refluxed with $80 \% \mathrm{EtOH}(16 \mathrm{~L})$ for $72 \mathrm{~h}$, and the ethanol extract was concentrated under vacuum to yield a dark green residue $(290.0 \mathrm{~g})$. The residue $(290.0 \mathrm{~g})$ was suspended in $\mathrm{H}_{2} \mathrm{O}(2.0 \mathrm{~L})$, and the aqueous layer was partitioned with $n$-hexane, ethyl acetate, and $n$-butanol. The ethyl acetate layer ( $54.9 \mathrm{~g}$ ) was subjected to silica gel column eluted with $\mathrm{CH}_{2} \mathrm{Cl}_{2}: \mathrm{MeOH}(8: 1 \rightarrow 2: 1)$ to obtain four fractions (5A-5D). Fraction $5 \mathrm{~B}$ was chromatographed on silica

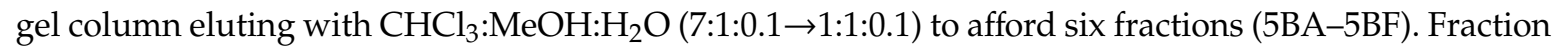


5BA was chromatographed on C-18 column eluting with $\mathrm{MeOH}: \mathrm{H}_{2} \mathrm{O}(1: 3 \rightarrow 1: 1)$ to isolate compound 1 (304.4 mg). Fraction 5BB was chromatographed on silica gel column eluting with $\mathrm{CHCl}_{3}: \mathrm{MeOH}: \mathrm{H}_{2} \mathrm{O}$ (4:1:0.1 $\rightarrow 1: 1: 0.1)$ to obtain two fractions (5BBA-5BBB). Fraction 5BBB was subjected to C-18 column eluting with $\left(\mathrm{CH}_{3}\right)_{2} \mathrm{CO}: \mathrm{MeOH}: \mathrm{H}_{2} \mathrm{O}(1: 1: 3 \rightarrow 1: 1: 1)$ to isolate compound 3 (69.0 mg). Fraction 5BD was subjected to $\mathrm{C}-18$ column eluted with $\left(\mathrm{CH}_{3}\right)_{2} \mathrm{CO}: \mathrm{H}_{2} \mathrm{O}(1: 3 \rightarrow 1: 1)$ to obtain compound $2(128.5 \mathrm{mg})$. Fraction 5BE was subjected to C-18 column using $\mathrm{MeOH}: \mathrm{H}_{2} \mathrm{O}(1: 3 \rightarrow 1: 1)$ to afford compound 4 (230.2 $\mathrm{mg})$. The fraction $5 \mathrm{BF}$ was subjected to $\mathrm{C}-18$ column eluted with $\mathrm{MeOH}: \mathrm{H}_{2} \mathrm{O}(1: 4 \rightarrow 1: 1)$ to obtain compound $5(52.7 \mathrm{mg})$. Fraction $5 \mathrm{C}$ was subjected to $\mathrm{C}-18$ column eluted with $\left(\mathrm{CH}_{3}\right)_{2} \mathrm{CO}: \mathrm{H}_{2} \mathrm{O}(1: 5 \rightarrow 1: 1)$ to obtain compound 7 (110.5 mg). Fraction 5D was chromatographed on C-18 column eluting with $\left(\mathrm{CH}_{3}\right)_{2} \mathrm{CO}: \mathrm{H}_{2} \mathrm{O}(1: 4 \rightarrow 1: 1)$ to obtain compound $6(67.5 \mathrm{mg})$.

\section{Compound 1}

White powder; ESI-MS: $m / z 373.2[\mathrm{M}-\mathrm{H}]^{-} ;{ }^{1} \mathrm{H}-\mathrm{NMR}$ (methanol- $\left.d_{4}, 400 \mathrm{MHz}\right): \delta 6.02(1 \mathrm{H}, \mathrm{d}, J=2.8 \mathrm{~Hz}$, H-5), $5.97\left(2 \mathrm{H}, \mathrm{d}, J=2.1 \mathrm{~Hz}, \mathrm{H}-6^{\prime \prime}, \mathrm{H}-2^{\prime \prime}\right), 5.89\left(1 \mathrm{H}, \mathrm{t}, J=2.1 \mathrm{~Hz}, \mathrm{H}-4^{\prime \prime}\right), 5.86\left(2 \mathrm{H}, \mathrm{s}, \mathrm{H}-5^{\prime}, \mathrm{H}-3^{\prime}\right), 5.72(1 \mathrm{H}$, d, $J=2.8 \mathrm{~Hz}, \mathrm{H}-3) .{ }^{13} \mathrm{C}-\mathrm{NMR}$ (methanol- $\left.d_{4}, 100 \mathrm{MHz}\right): \delta 162.5\left(\mathrm{C}-1^{\prime \prime}\right), 160.4\left(\mathrm{C}-3^{\prime \prime}, \mathrm{C}-5^{\prime \prime}\right), 156.5\left(\mathrm{C}-4^{\prime}\right)$, 156.2 (C-4), 153.8 (C-6), 152.7 (C-2), 152.2 (C-6', C-2'), 125.7 (C-1), 124.7 (C-1'), 98.0 (C-3), 97.5 (C-4"), $96.2\left(\mathrm{C}-5^{\prime}, \mathrm{C}-3^{\prime}\right), 95.4\left(\mathrm{C}-2^{\prime \prime}, \mathrm{C}-6^{\prime \prime}\right), 95.0(\mathrm{C}-5)$.

\section{Compound 2}

Light brown powder; ESI-MS: $m / z 371.2[\mathrm{M}-\mathrm{H}]^{-} ;{ }^{1} \mathrm{H}-\mathrm{NMR}$ (DMSO- $\left.d_{6}, 300 \mathrm{MHz}\right): \delta 9.54$ (1H, s, OH-9), $9.48(1 \mathrm{H}, \mathrm{s}, \mathrm{OH}-4), 9.21(2 \mathrm{H}, \mathrm{s}, \mathrm{OH}-2, \mathrm{OH}-7), 9.17\left(2 \mathrm{H}, \mathrm{s}, \mathrm{OH}-3^{\prime}, \mathrm{OH}-5^{\prime}\right), 6.14(1 \mathrm{H}, \mathrm{s}, \mathrm{H}-3), 5.97(1 \mathrm{H}, \mathrm{d}$, $J=2.5 \mathrm{~Hz}, \mathrm{H}-8), 5.81\left(1 \mathrm{H}, \mathrm{d}, J=1.7 \mathrm{~Hz}, \mathrm{H}-4^{\prime}\right), 5.80(1 \mathrm{H}, \mathrm{d}, J=2.5 \mathrm{~Hz}, \mathrm{H}-6), 5.72\left(2 \mathrm{H}, \mathrm{d}, J=1.7 \mathrm{~Hz}, \mathrm{H}-2^{\prime}\right.$, H-6' $) .{ }^{13} \mathrm{C}-\mathrm{NMR}$ (DMSO- $\left.d_{6}, 75 \mathrm{MHz}\right): \delta 160.4\left(\mathrm{C}-1^{\prime}\right), 158.9$ (C-3', C-5'), 153.1 (C-7), 146.1 (C-9), 146.0 (C-2), 142.6 (C-5a), 142.0 (C-4), 137.2 (C-10a), 123.2 (C-1), 122.7 (C-9a), 122.2 (C-4a), 98.5 (C-8), 98.2 (C-3), $96.3\left(\mathrm{C}-4^{\prime}\right), 93.9(\mathrm{C}-6), 93.7\left(\mathrm{C}-2^{\prime}, \mathrm{C}-6^{\prime}\right)$.

\section{Compound 3}

Light brown powder; ESI-MS: $m / z$ 495.2 [M - H] $]^{-} ;{ }^{1} \mathrm{H}$ NMR (DMSO- $d_{6}, 400 \mathrm{MHz}$ ): $\delta 9.52$ (1H, s, OH-9), $9.41(1 \mathrm{H}, \mathrm{s}, \mathrm{OH}-4), 9.19(1 \mathrm{H}, \mathrm{s}, \mathrm{OH}-7), 9.13\left(2 \mathrm{H}, \mathrm{s}, \mathrm{OH}-3^{\prime}, \mathrm{OH}-5^{\prime}\right), 9.08\left(2 \mathrm{H}, \mathrm{s}, \mathrm{OH}-2^{\prime \prime}, \mathrm{OH}-6^{\prime \prime}\right), 8.98(1 \mathrm{H}$, s, OH-4"), $5.98(1 \mathrm{H}, \mathrm{d}, J=2.2 \mathrm{~Hz}, \mathrm{H}-8), 5.87\left(2 \mathrm{H}, \mathrm{d}, J=1.8 \mathrm{~Hz}, \mathrm{H}-2^{\prime}, \mathrm{H}-6^{\prime}\right), 5.85$ (2H, s, H-3", H-5"'), $5.84\left(1 \mathrm{H}, \mathrm{t}, J=1.8 \mathrm{~Hz}, \mathrm{H}-4^{\prime}\right), 5.83(1 \mathrm{H}, \mathrm{s}, \mathrm{H}-3), 5.80(1 \mathrm{H}, \mathrm{d}, J=2.2 \mathrm{~Hz}, \mathrm{H}-5) .{ }^{13} \mathrm{C}$ NMR (DMSO- $d_{6}$, $100 \mathrm{MHz}): \delta 160.5$ (C-1'), 158.9 (C-3', C-5'), 154.9 (C-4"), 153.2 (C-7), 151.3 (C-2", C-6"), 147.9 (C-2), 146.3 (C-9), 142.6 (C-5a), 141.7 (C-4), 137.2 (C-10a), 124.4 (C-4a), 122.7 (C-1), 122.0 (C-9a), 98.7 (C-8), $96.4\left(\mathrm{C}-4^{\prime}\right), 96.0(\mathrm{C}-3), 94.9\left(\mathrm{C}-3^{\prime \prime}, \mathrm{C}-5^{\prime \prime}\right), 94.1\left(\mathrm{C}-2^{\prime}, \mathrm{C}-6^{\prime}\right), 93.9$ (C-6).

\section{Compound 4}

Amorphous powder; ESI-MS: m/z 601.2 [M - H] $]^{-}$; 1H-NMR (DMSO-d6, $300 \mathrm{MHz}$ ): $\delta 10.14$ (1H, s, OH-14), $9.87(1 \mathrm{H}, \mathrm{s}, \mathrm{OH}-4), 9.85(1 \mathrm{H}, \mathrm{s}, \mathrm{OH}-10), 9.44(1 \mathrm{H}, \mathrm{s}, \mathrm{OH}-2), 9.21\left(2 \mathrm{H}, \mathrm{s}, \mathrm{OH}-3^{\prime}, \mathrm{OH}-5^{\prime}\right), 9.18$ (2H, s, OH-3", OH-5"), $8.22(1 \mathrm{H}, \mathrm{s}, \mathrm{OH}-8), 6.73(1 \mathrm{H}, \mathrm{s}, \mathrm{H}-13), 6.44(1 \mathrm{H}, \mathrm{s}, \mathrm{H}-9), 6.31(1 \mathrm{H}, \mathrm{s}, \mathrm{H}-3)$, $5.84\left(2 \mathrm{H}, \mathrm{d}, J=1.8 \mathrm{~Hz}, \mathrm{H}-4^{\prime}, \mathrm{H}-4^{\prime \prime}\right), 5.78\left(2 \mathrm{H}, \mathrm{d}, J=1.8 \mathrm{~Hz}, \mathrm{H}-2^{\prime}, \mathrm{H}-6^{\prime}\right), 5.73\left(2 \mathrm{H}, \mathrm{d}, J=1.8 \mathrm{~Hz}, \mathrm{H}-2^{\prime \prime}\right.$, H-6"). ${ }^{13}$ C-NMR (DMSO- $\left.d_{6}, 75 \mathrm{MHz}\right): \delta 160.2\left(\mathrm{C}-1^{\prime}\right), 160.0$ (C-1"), 159.0 (C-3", C-5"), 158.8 (C-3', C-5'), 150.8 (C-12a), 150.4 (C-10), 149.5 (C-11a), 147.0 (C-2), 146.5 (C-8), 144.8 (C-14), 142.0 (C-4), 136.8 (C-15a), 134.0 (C-5a), 126.4 (C-14a), 122.6 (C-4a), 122.5 (C-1), 120.1 (C-11), 103.4 (C-7), 103.2 (C-6), 99.1 (C-9), 98.3 (C-3), $96.5\left(\mathrm{C}-4^{\prime \prime}\right), 96.4\left(\mathrm{C}-4^{\prime}\right), 94.8$ (C-13), 93.7 (C-2', C-6'), 93.5 (C-2", C-6").

\section{Compound 5}

Amorphous powder; ESI-MS: $m / z$ 865.3 [M - H] ${ }^{-} ;{ }^{1} \mathrm{H}$ NMR (DMSO- $\left.d_{6}, 400 \mathrm{MHz}\right): \delta 9.29$ (1H, s, OH-9), $9.26\left(1 \mathrm{H}, \mathrm{s}, \mathrm{OH}-9^{\prime}\right), 9.15\left(5 \mathrm{H}, \mathrm{s}, \mathrm{OH}-3^{\prime \prime}, \mathrm{OH}-5^{\prime \prime}, \mathrm{OH}-3^{\prime \prime \prime}, \mathrm{OH}-5^{\prime \prime \prime}, \mathrm{OH}-2^{\prime}\right), 9.09(\mathrm{IH}, \mathrm{s}, \mathrm{OH}-4), 9.07(1 \mathrm{H}, \mathrm{s}$,

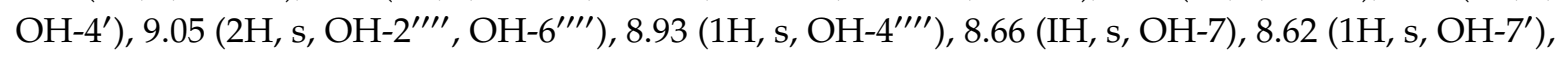


$6.08\left(1 \mathrm{H}, \mathrm{s}, \mathrm{H}-3^{\prime}\right), 6.06(1 \mathrm{H}, \mathrm{s}, \mathrm{H}-8), 6.04\left(1 \mathrm{H}, \mathrm{s}, \mathrm{H}-8^{\prime}\right), 5.90\left(2 \mathrm{H}, \mathrm{s}, J=2.1 \mathrm{~Hz}, \mathrm{H}-2^{\prime \prime}, \mathrm{H}-6^{\prime \prime}\right), 5.83(1 \mathrm{H}, \mathrm{d}$, $\left.J=2.1 \mathrm{~Hz}, \mathrm{H}-4^{\prime \prime}\right), 5.83\left(2 \mathrm{H}, \mathrm{s}, \mathrm{H}-3^{\prime \prime \prime}, \mathrm{H}-5^{\prime \prime \prime}\right), 5.80(1 \mathrm{H}, \mathrm{s}, \mathrm{H}-3), 5.79\left(1 \mathrm{H}, \mathrm{d}, J=3.0 \mathrm{~Hz}, \mathrm{H}-4^{\prime \prime \prime \prime}\right), 5.74(2 \mathrm{H}$, $\left.\mathrm{d}, J=3.0 \mathrm{~Hz}, \mathrm{H}-2^{\prime \prime \prime \prime}, \mathrm{H}-6^{\prime \prime \prime \prime}\right) .{ }^{13} \mathrm{C}$ NMR (DMSO- $\left.d_{6}, 100 \mathrm{MHz}\right): \delta 160.6$ (C-1", C-1'"'), 158.9 (C-3", C-3'"',

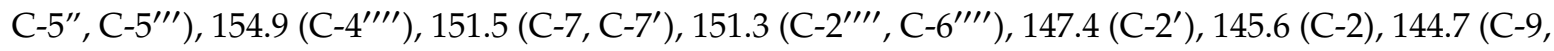

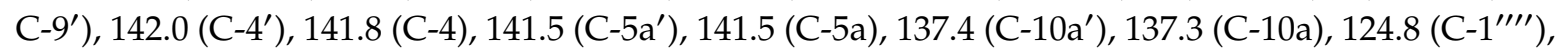
123.8 (C-1"), 122.9 (C-9a, C-9a'), 122.5 (C-1), 122.1 (C-4a, C-4a'), 99.8 (C-6'), 99.7 (C-6), 98.0 (C-8, C-8'),

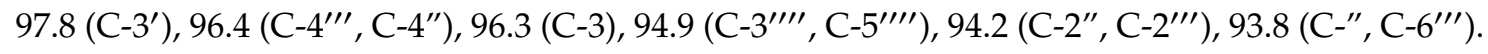

\section{Compound 6}

Brown amorphous powder; ESI-MS: $m / z 741.2[\mathrm{M}-\mathrm{H}]^{-} ;{ }^{1} \mathrm{H}$ NMR (DMSO- $d_{6}, 400 \mathrm{MHz}$ ): $\delta 9.46(1 \mathrm{H}, \mathrm{s}$, OH-2'), 9.28 (1H, s, OH-9'), 9.17 (3H, s, OH-3"' , OH-5"' , OH-4'), $9.12\left(3 \mathrm{H}, \mathrm{s}, \mathrm{OH}-3^{\prime \prime}, \mathrm{OH}-5^{\prime \prime}, \mathrm{OH}-2\right)$, $9.07(1 \mathrm{H}, \mathrm{s}, \mathrm{OH}-4), 8.76\left(1 \mathrm{H}, \mathrm{s}, \mathrm{OH}-7^{\prime}\right), 8.65(1 \mathrm{H}, \mathrm{s}, \mathrm{OH}-7), 7.90(1 \mathrm{H}, \mathrm{s}, \mathrm{OH}-9), 6.19(1 \mathrm{H}, \mathrm{d}, \mathrm{H}-8), 6.10$ $\left(1 \mathrm{H}, \mathrm{s}, \mathrm{H}-3^{\prime}\right), 6.06(1 \mathrm{H}, \mathrm{s}, \mathrm{H}-3), 5.97\left(1 \mathrm{H}, \mathrm{s}, \mathrm{H}-6^{\prime}\right), 5.81\left(2 \mathrm{H}, \mathrm{d}, J=2.0 \mathrm{~Hz}, \mathrm{H}-4^{\prime \prime}, \mathrm{H}-4^{\prime \prime \prime}\right), 5.78$ (2H, d, $\left.J=2.0 \mathrm{~Hz}, \mathrm{H}-2^{\prime \prime \prime}, \mathrm{H}-6^{\prime \prime \prime}\right), 5.76\left(2 \mathrm{H}, \mathrm{d}, J=2.0 \mathrm{~Hz}, \mathrm{H}-2^{\prime \prime}, \mathrm{H}-6^{\prime \prime}\right) .{ }^{13} \mathrm{C}$ NMR (DMSO- $d_{6}, 100 \mathrm{MHz}$ ): $\delta 160.4$ (C-1", C-1"'), 158.8 (C-3", C-3'"', C-5", C-5"'), 151.7 (C-7), 151.5 (C-7'), 145.8 (C-2), 145.4 (C-2'), 144.6 (C-9), 144.3 (C-9'), 141.8 (C-4), 141.6 (C-4'), 141.2 (C-5a), 140.9 (C-5a'), 137.2 (C-10a), 137.2 (C-10a'), 123.5 (C-1), $123.2\left(\mathrm{C}-1^{\prime}\right), 123.0$ (C-9a), 122.8 (C-9a'), 122.3 (C-4a), $121.8\left(\mathrm{C}-4 \mathrm{a}^{\prime}\right), 104.1\left(\mathrm{C}-8^{\prime}\right), 99.3$ (C-6), $98.0(\mathrm{C}-8), 96.1\left(\mathrm{C}-4^{\prime \prime}\right), 96.0\left(\mathrm{C}-4^{\prime \prime \prime}\right), 93.8\left(\mathrm{C}-2^{\prime \prime \prime}, \mathrm{C}-6^{\prime \prime \prime}\right), 93.7\left(\mathrm{C}-2^{\prime \prime}, \mathrm{C}-6^{\prime \prime}\right), 93.6\left(\mathrm{C}-6^{\prime}\right)$.

\section{Compound 7}

Brown amorphous powder; ESI-MS: $m / z 741.2[\mathrm{M}-\mathrm{H}]^{-} ;{ }^{1} \mathrm{H}$ NMR (DMSO- $\left.d_{6}, 300 \mathrm{MHz}\right): \delta 9.46(1 \mathrm{H}, \mathrm{s}$, OH-4), 9.18 (1H, s, OH-2), $9.13\left(2 \mathrm{H}, \mathrm{s}, \mathrm{OH}-3^{\prime}, \mathrm{OH}^{-5} 5^{\prime}\right), 8.77$ (1H, s, OH-9), $7.91(1 \mathrm{H}, \mathrm{s}, \mathrm{OH}-7), 6.18(1 \mathrm{H}$, $\mathrm{s}, \mathrm{H}-3), 5.98(1 \mathrm{H}, \mathrm{s}, \mathrm{H}-6), 5.81\left(1 \mathrm{H}, \mathrm{t}, J=1.8 \mathrm{~Hz}, \mathrm{H}-4^{\prime}\right), 5.76\left(2 \mathrm{H}, \mathrm{d}, J=1.8 \mathrm{~Hz}, \mathrm{H}-2^{\prime}, \mathrm{H}-6^{\prime}\right) .{ }^{13} \mathrm{C} \mathrm{NMR}$ (DMSO- $d_{6}, 75$ MHz): $\delta 160.9$ (C-1'), 159.2 (C-3', C-5'), 152.2 (C-7), 146.3 (C-2), 145.1 (C-9), 142.3 (C-4), 141.8 (C-5a), 137.7 (C-10a), 123.8 (C-1), 123.6 (C-9a), 122.9 (C-4a), 104.7 (C-8), 98.6 (C-3), 96.7 (C-4'), $94.4\left(\mathrm{C}-2^{\prime}, \mathrm{C}-6^{\prime}\right), 94.3(\mathrm{C}-6)$.

\subsection{Tyrosinase Assay}

To evaluate inhibitory activity on tyrosinase with isolated compounds, $130 \mu \mathrm{L}$ of tyrosinase in $0.05 \mathrm{mM}$ phosphate buffer ( $\mathrm{pH}$ 6.8) was divided in 96 well plates [19]. $20 \mu \mathrm{L}$ of compound concentrations ranging from 1-0.015 mM was added. $50 \mu \mathrm{L}$ of $1.5 \mathrm{mM}$ L-tyrosine in phosphate buffer was diluted into the mixture for calculating the inhibitory activity. $50 \mu \mathrm{L}$ of $10-0.62 \mathrm{mM}$ L-tyrosine in buffer was added to analyze initial velocity $\left(v_{0}\right)$. After starting their reaction for $20 \mathrm{~min}$, an amount of the product was detected at UV-vis $475 \mathrm{~nm}$. The inhibitory activity was analyzed according to Equation (1)

$$
\text { Inhibitory activity rate }(\%)=[(\Delta \mathrm{C}-\Delta \mathrm{S}) / \Delta \mathrm{C}] \times 100
$$

\subsection{Slow-Binding Inhibition Analysis}

The progress curves were calculated to Equation (2) by Morrison according to time course for $420 \mathrm{~s}$.

$$
[\mathrm{P}]=v_{s} t+\left[\left(v_{0}-v_{\mathrm{s}}\right) / k_{\mathrm{obs}}\left(1-\mathrm{e}^{-k_{\text {obst }}}\right)\right]
$$

where $t$ is time, $[\mathrm{P}]$ is product intensity, $v_{0}$ and $v_{\mathrm{S}}$ are the initial and steady-state reaction velocities, and $k_{\mathrm{obs}}$ is the apparent first-order rate concentration.

$$
\begin{gathered}
k_{\mathrm{obs}}=k_{4}+k_{3}[I] \\
K_{i}^{a p p}=k_{4} / k_{3}
\end{gathered}
$$


This is a linear Equation (3) for slow-binding mechanism A. $K_{i}^{a p p}$ is the apparent value of an inhibitor by Equation (4). Where [I] is inhibitor concentration.

$$
k_{\mathrm{obs}}=k_{6}+\left[\left(k_{5} \times[I]\right) /\left(K_{i}^{a p p}+[I]\right)\right.
$$

Equation (5) of hyperbola is for the calculation of rate constant in slow-binding mechanism B.

\subsection{Molecular Docking}

This simulation was performed as described previously. The 3D structure of protein coded with 2Y9X was achieved from RCSB homepage [22]. This was added hydrogen atoms and assigned with gasteiger charges by using Autodocktools. Ligands were built and minimized energy by MM2 with Chem3D Pro. They were established the grid containing activity site with number of points (X: 80, Y: 80, Z: 80). DPS file was contained to set up Lamarckian genetic algorithm for docking of the ligand into receptor (runs 50 and the maximum number was set as long). Their results were represented with Chimera (version 3.13.1, San Francisco, CA, USA) and LigPlot (version 4.5.3, Cambridge, UK).

\section{Conclusions}

Seven phlorotannins (1-7) from E. cava were isolated using column chromatography and their structures were identified by spectral analysis. Of these, compounds 3 and 5 potentially inhibited the catalytic reaction of tyrosinase by blocking the entrance to the active site. Moreover, compounds 3 and 5 altered the turnover rate from substrate to the enzyme product over time. The former underwent simple reversible slow binding with the enzyme, while the latter initially formed a complex (EI) with the enzyme quickly, followed by an isomerization step to create a new enzyme-type complex (E*I). Phlorotannins containing flexible phloroglucinol to eckol backbone were found the facts bound easily into active site of tyrosinase. The findings suggested the phloroglucinol may be key moiety for induced fit of inhibitor with enzyme flexibility. The molecular simulation study indicated that the two inhibitors were docked at the active site of tyrosinase with AutoDock scores or -6.59 and $-6.68 \mathrm{kcal} / \mathrm{mol}$, respectively. Both compounds used the amino acids His 85 and Asn 260 to maintain their connection with tyrosinase. These findings imply that 2-phloroeckol (3) and 2-O-(2,4,6-trihydroxyphenyl)-6,6'-bieckol (5) might inhibit the production of melanin products.

Author Contributions: Y.H.K. and S.Y.Y. participated in the research design. S.P. and J.S.P. conducted the experiments. J.H.K. and S.L. contributed to the writing of the manuscript and the experiments.

Funding: This research was supported by Basic Science Research Program through the National Research Foundation of Korea (NRF) funded by the Ministry of Education, Science and Technology (NRF-2018R1A6A 3A11047338).

Conflicts of Interest: The authors declare no conflict of interest.

\section{References}

1. Şöhretoğlu, D.; Sari, S.; Barut, B.; Özel, A. Tyrosinase inhibition by some flavonoids: Inhibitory activity, mechanism by in vitro and in silico studies. Bioorg. Chem. 2018, 81, 168-174. [CrossRef] [PubMed]

2. Bukhari, S.N.A.; Jantan, I.; Tan, O.U.; Sher, M.; Naeem-ul-Hassan, M.; Qin, H.-L. Biological activity and molecular docking studies of curcumin-related $\alpha, \beta$-unsaturated carbonyl-based synthetic compounds as anticancer agents and mushroom tyrosinase inhibitors. J. Agric. Food Chem. 2014, 62, 5538-5547. [CrossRef] [PubMed]

3. Ha, Y.M.; Park, Y.J.; Lee, J.Y.; Park, D.; Choi, Y.J.; Lee, E.K.; Kim, J.M.; Kim, J.-A.; Park, J.Y.; Lee, H.J.; et al. Design, synthesis and biological evaluation of 2-(substituted phenyl)thiazolidine-4-carboxylic acid derivatives as novel tyrosinase inhibitors. Biochimie 2012, 94, 533-540. [CrossRef] [PubMed]

4. Wang, Y.; Zhang, G.; Yan, J.; Gong, D. Inhibitory effect of morin on tyrosinase: Insights from spectroscopic and molecular docking studies. Food Chem. 2014, 163, 226-233. [CrossRef] [PubMed] 
5. Lan, W.-C.; Tzeng, C.-W.; Lin, C.-C.; Yen, F.-L.; Ko, H.-H. Prenylated flavonoids from Artocarpus altilis: Antioxidant activities and inhibitory effects on melanin production. Phytochemistry 2013, 89, 78-88. [CrossRef] [PubMed]

6. Wang, H.-M.; Chen, C.-Y.; Chen, C.-Y.; Ho, M.-L.; Chou, Y.-T.; Chang, H.-C.; Lee, C.-H.; Wang, C.-Z.; Chu, I.-M. (-)-N-Formylanonaine from Michelia alba as a human tyrosinase inhibitor and antioxidant. Bioorg. Med. Chem. 2010, 18, 5241-5247. [CrossRef] [PubMed]

7. Zolghadri, S.; Bahrami, A.; Khan, M.T.H.; Munoz-Munoz, J.; Garcia-Molina, F.; Garcia-Canovas, F.; Saboury, A.A. A comprehensive review on tyrosinase inhibitors. J. Enzyme Inhib. Med. Chem. 2019, 34, 279-309. [CrossRef]

8. Lee, J.; Jun, M. Dual BACE1 and cholinesterase inhibitory effects of phlorotannins from Ecklonia cava-An in vitro and in silico study. Mar. Drugs 2019, 17, 91. [CrossRef]

9. Yang, H.; Lee, S.Y.; Lee, S.R.; Pyun, B.-J.; Kim, H.J.; Lee, Y.H.; Kwon, S.W.; Suh, D.H.; Lee, C.H.; Hong, E.-J.; et al. Therapeutic effect of Ecklonia cava extract in letrozole-induced polycystic ovary syndrome rats. Front. Pharmacol. 2018, 9, 1325. [CrossRef]

10. Oh, S.; Son, M.; Lee, H.S.; Kim, H.-S.; Jeon, Y.-J.; Byun, K. Protective effect of pyrogallol-phloroglucinol6,6-bieckol from Ecklonia cava on monocyte-associated vascular dysfunction. Mar. Drugs 2018, 16, 441. [CrossRef]

11. Yang, Y.-I.; Woo, J.-H.; Seo, Y.-J.; Lee, K.-T.; Lim, Y.; Choi, J.-H. Protective effect of brown alga not italic phlorotannins against hyperinflammatory responses in lipopolysaccharide-induced sepsis models. J. Agric. Food Chem. 2016, 64, 570-578. [CrossRef] [PubMed]

12. Kang, H.S.; Kim, H.R.; Byun, D.S.; Son, B.W.; Nam, T.J.; Choi, J.S. Tyrosinase inhibitors isolated from the edible brown alga Eckloina stolonifera. Arch. Pharmcal. Res. 2004, 27, 1226-1232. [CrossRef]

13. Yoon, N.Y.; Eom, T.-K.; Kim, M.-M.; Kim, S.-K. Inhibitory effect of phlorotannins isolated from Ecklonia cava on mushroom tyrosinase activity and melanin formation in mouse B16F10 melanoma cells. J. Agric. Food Chem. 2009, 57, 4124-4129. [CrossRef] [PubMed]

14. Wijesinghe, W.A.J.P.; Ko, S.-C.; Jeon, Y.-J. Effect of phlorotannins isolated from Ecklonia cava on angiotensin enzyme (ACE) inhibitory activity. Nutr. Res. Pract. 2011, 5, 93-100. [CrossRef] [PubMed]

15. Lee, J.H.; Ko, J.Y.; Oh, J.Y.; Kim, C.Y.; Lee, H.J.; Kim, J.; Jeon, Y.J. Preparative isolation and purification of phlorotannins from Ecklonia cava using centrifugal partition chromatography by one-step. Food Chem. 2014, 158, 433-437. [CrossRef]

16. Park, J.Y.; Kim, J.H.; Kwon, J.M.; Kwon, H.J.; Jeong, H.J.; Kim, Y.M.; Kim, D.; Lee, W.S.; Ryu, Y.B. Dieckol, a SARS-CoV 3CL pro inhibitor, isolated from the edible brown algae Ecklonia cava. Bioorg. Med. Chem. 2013, 21, 3730-3737. [CrossRef] [PubMed]

17. Fukuyama, Y.; Kodama, M.; Miura, I.; Kinzyo, Z.; Mori, H.; Nakayama, Y.; Takahashi, M. Anti-plasmin Inhibitor. V.: Structures of novel dimeric eckols isolated from the brown alga Ecklonia kurome OKAMURA. Chem. Pharm. Bull. 1989, 37, 2438-2440. [CrossRef]

18. Sugiura, Y.; Matsuda, K.; Yamada, Y.; Nishikawa, M.; Shioya, K.; Katsuzaki, H.; Imai, K.; Amano, H. Anti-allergic phlorotannins from the edible brown alga, Eisenia arborea. Food Sci. Technol. Res. 2007, 13, 54-60. [CrossRef]

19. Kang, S.-M.; Heo, S.-J.; Kim, K.-N.; Yang, S.-H.; Kim, A.-D.; Jeon, Y.-J. Molecular docking studies of a phlorotannin, dieckol isolated from Eckloina cava with tyrosinase inhibitory activity. Bioorg. Med. Chem. 2012, 20, 311-316. [CrossRef]

20. Masson, P.; Lushchekina, S.V. Slow-binding inhibition of cholinesterase, pharmacological and toxicological relevance. Arch. Biochem. Biophys. 2016, 593, 60-68. [CrossRef]

21. Menon, V.; Rao, M. Slow-tight binding inhibition of pepsin by an aspartic protease inhibitor from Streptomyces sp. MBR04. Inter. J. Biol. Macromol. 2012, 51, 165-174. [CrossRef] [PubMed]

22. Xiong, S.-L.; Lim, G.T.; Yin, S.-J.; Lee, J.; Si, Y.-X.; Yang, J.-M.; Park, Y.-D.; Qian, G.-Y. The inhibitory effect of pyrogallol on tyrosinase activity and structure: Integration study of inhibition kinetics with molecular dynamics simulation. Int. J. Biol. Macromol. 2019, 121, 463-471. [CrossRef] [PubMed]

(C) 2019 by the authors. Licensee MDPI, Basel, Switzerland. This article is an open access article distributed under the terms and conditions of the Creative Commons Attribution (CC BY) license (http://creativecommons.org/licenses/by/4.0/). 